\title{
Breath-Hold Blood Oxygen Level-Dependent MRI: A Tool for the Assessment of Cerebrovascular Reserve in Children with Moyamoya Disease
}

\author{
(D) N. Dlamini, (DP. Shah-Basak, (D). Leung, (D). Kirkham, (D) M. Shroff, (D)A. Kassner, (D) A. Robertson, (D) Pirks, (D). Westmacott, \\ (D) G. deVeber, and (DW. Logan

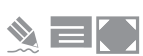

\begin{abstract}
BACKGROUND AND PURPOSE: There is a critical need for a reliable and clinically feasible imaging technique that can enable prognostication and selection for revascularization surgery in children with Moyamoya disease. Blood oxygen level-dependent MR imaging assessment of cerebrovascular reactivity, using voluntary breath-hold hypercapnic challenge, is one such simple technique. However, its repeatability and reliability in children with Moyamoya disease are unknown. The current study sought to address this limitation.
\end{abstract}

MATERIALS AND METHODS: Children with Moyamoya disease underwent dual breath-hold hypercapnic challenge blood oxygen leveldependent MR imaging of cerebrovascular reactivity in the same MR imaging session. Within-day, within-subject repeatability of cerebrovascular reactivity estimates, derived from the blood oxygen level-dependent signal, was computed. Estimates were associated with demographics and intellectual function. Interrater reliability of a qualitative and clinically applicable scoring scheme was assessed.

RESULTS: Twenty children (11 males; $12.1 \pm 3.3$ years) with 30 MR imaging sessions (60 MR imaging scans) were included. Repeatability was "good" on the basis of the intraclass correlation coefficient $(0.70 \pm 0.19)$. Agreement of qualitative scores was "substantial" ( $\kappa=0.711)$, and intrarater reliability of scores was "almost perfect" ( $\kappa=0.83$ and 1). Younger participants exhibited lower repeatability $(P=.027)$. Repeatability was not associated with cognitive function $(P>.05)$. However, abnormal cerebrovascular reactivity was associated with slower processing speed $(P=.015)$.

CONCLUSIONS: Breath-hold hypercapnic challenge blood oxygen level-dependent MR imaging is a repeatable technique for the assessment of cerebrovascular reactivity in children with Moyamoya disease and is reliably interpretable for use in clinical practice. Standardization of such protocols will allow further research into its application for the assessment of ischemic risk in childhood cerebrovascular disease.

ABBREVIATIONS: $\mathrm{BH}=$ breath-hold; $\mathrm{BOLD}=$ blood oxygen level-dependent; $\mathrm{CV}$ = coefficient of variation; $\mathrm{CVR}=$ cerebrovascular reactivity; $\mathrm{ICC}=$ intraclass correlation coefficient; $\mathrm{SCD}=$ sickle cell disease

A rterial ischemic stroke remains a major cause of morbidity and mortality in children worldwide. The incidence ranges from 2 to 13 per 100,000 person-years in developed countries. ${ }^{1,2}$

Received February 15, 2018; accepted after revision June 10.

From the Division of Neurology (N.D., G.d.V., W.L.), Neurosciences and Mental Health Program (N.D.), Child Health Evaluative Sciences Program (N.D., A.R., G.d.V.), Translational Medicine (J.L., A.K.), Diagnostic Imaging (P.S.-B., M.S.), Department of Neurosurgery (P.D.), and Department of Neuropsychology (R.W.), The Hospital for Sick Children, Toronto, Ontario, Canada; Institute of Medical Science (N.D., G.d.V.) and Department of Medical Imaging (A.K.), University of Toronto, Toronto, Ontario, Canada; Rotman Research Institute (P.S.-B.), Baycrest, Toronto, Ontario, Canada; and Developmental Neurosciences (N.D., F.K.), University College London, Great Ormond Street Institute of Child Health, London, UK.

Please address correspondence to Nomazulu Dlamini, Division of Neurology, The Hospital for Sick Children, 555 University Ave, Toronto, M5G 1X8, Canada, e-mail: nomazulu.dlamini@sickkids.ca

$\equiv$ Indicates article with supplemental on-line tables.

Indicates article with supplemental on-line photos.

http://dx.doi.org/10.3174/ajnr.A5739
Childhood risk factors are multiple and include cardiac disease, sickle cell disease (SCD), arteriopathy, and infection. ${ }^{3-5}$ More than two-thirds of previously healthy children presenting with their first arterial ischemic stroke have a steno-occlusive arteriopathy, the presence of which predicts recurrence and outcome. ${ }^{6-8}$ Moyamoya disease is a major arteriopathy of childhood. It is a progressive steno-occlusive arteriopathy that typically affects the anterior circulation arteries of the circle of Willis. It confers a life-long risk of recurrent stroke and neurologic injury, and it is associated with early death due to chronic cerebral hypoperfusion and thrombotic vaso-occlusion. ${ }^{9-11}$ However, the pathology of the primary arteriopathy is poorly understood.

A network of lenticulostriate collaterals develops to bypass the primary steno-occlusive arteriopathy, and vasodilation at the level of the capillary bed occurs to maintain cerebral blood flow, resulting in a reduction of cerebrovascular reserve. ${ }^{12}$ Studies in adults with arteriopathy suggest that impairment of cerebrovas- 
cular reserve is associated with an approximate 4-fold increased risk of developing stroke or transient ischemic attacks. ${ }^{13}$ In vivo assessment of cerebrovascular reserve can be performed by measurement of cerebrovascular reactivity (CVR), defined as a change in CBF in response to vasoactive stimuli such as carbon dioxide. In the context of steno-occlusive arteriopathy and maximal microvascular vasodilation in response to falling $\mathrm{CBF}$, exhaustion of cerebrovascular reserve and vasodilatory capacity may result in paradoxical reductions in CBF and CVR following a vasodilating stimulus. ${ }^{12,14-16}$ This negative response, termed "steal," is an independent predictor of ischemic injury and stroke. ${ }^{12,14,15}$

Historically used techniques for assessing CBF and cerebrovascular reserve such as PET and SPECT require exposure to ionizing radiation and are hence undesirable. ${ }^{16}$ Blood oxygen leveldependent (BOLD) MR imaging is a widely used technique for the noninvasive imaging of dynamic changes in $\mathrm{CBF}$ at the local and global levels. BOLD MR imaging harnesses the paramagnetic properties of deoxyhemoglobin using clinically available $\mathrm{T} 2{ }^{*}$ gradient-echo MR imaging sequences and does not require intravenous contrast medium. Using carbon dioxide as a vasoactive stimulus, hypercapnic challenge BOLD MR imaging CVR (BOLD-CVR) can be used to generate high-spatial-resolution CVR maps. Thus, when cerebrovascular reserve is exhausted, negative or paradoxical reactivity on hypercapnic challenge BOLDCVR maps represents steal and provides a visual representation of ischemic risk and impending tissue demise. ${ }^{15}$

Hypercapnia induced by computer-controlled carbon dioxide stimulus delivery for BOLD-CVR experiments has been validated in both adults and children in research settings. ${ }^{17-21}$ In the only published study of the reliability of CVR measurements acquired in children (10 healthy children; mean age, $16.1 \pm 1.6$ years), intraclass correlations coefficients (ICCs) of within-day values were 0.857 and 0.895 in the GM and the WM, respectively. ${ }^{22}$ However, this method relies on specialized MR imaging-compatible equipment, limiting its applicability in clinical settings. Additionally, the use of nasal prongs or a facemask to deliver and monitor the carbon dioxide stimulus serves as a barrier for CVR acquisition in young children.

An alternative approach for performing CVR assessment is breath-hold $(\mathrm{BH})$, in which endogenous alveolar carbon dioxide naturally accumulates during short periods of voluntary apnea. Without the need for additional equipment and gas-delivery apparatus, $\mathrm{BH}$ is easier to implement and well-tolerated. ${ }^{22}$ The repeatability and reliability of BH-BOLD-CVR (BH-CVR) in highstroke-risk pediatric populations, however, remain unknown. The purpose of this study was to evaluate within-day, withinsubject repeatability, qualitative scoring, and interrater reliability of the scoring of $\mathrm{BH}-\mathrm{CVR}$ estimates. The relationship between clinical factors and intellectual function and $\mathrm{BH}$ performance and repeatability was also explored.

\section{MATERIALS AND METHODS Study Population}

Children 6 years of age or older diagnosed with cerebral vasculopathy consistent with a diagnosis of Moyamoya disease (idiopathic or syndromic) between 2010 and 2017 were recruited from the Hospital for Sick Children Toronto Stroke Registry. All pro- cedures were approved by our institutional Research Ethics Board, and informed written consent was obtained. Moyamoya disease was diagnosed if conventional or MR angiography demonstrated stenosis or occlusion of the distal internal carotid artery, the proximal middle cerebral artery, and/or the anterior cerebral artery with the appearance of lenticulostriate collaterals. Children with unilateral Moyamoya disease with collaterals (probable Moyamoya disease) were included. ${ }^{23}$ Children without a previously diagnosed condition were diagnosed as having Moyamoya disease or idiopathic Moyamoya disease, while those with a previously diagnosed condition such as neurofibromatosis type 1 or SCD were diagnosed as having Moyamoya syndrome. Children with non-Moyamoya arteriopathy and children unable to complete a repeat study were excluded.

\section{Breath-Hold Paradigm}

No minimum duration of $\mathrm{BH}$ was set for inclusion in the study. Children practiced the $\mathrm{BH}$ paradigm seated on a chair before entering the MR imaging scanner under the supervision of a research technologist. Each $\mathrm{BH}$ paradigm began with 10 seconds of normal breathing, followed by five 60 -second periods of breathholding and normal breathing (Fig $1 A$ ). Optimal BH duration was determined during practice in individual patients as the maximum number of seconds that they could hold their breath without discomfort. $\mathrm{BH}$ duration was subtracted from 60 seconds to calculate the within-cycle normal breathing duration. A 30-second rest period with normal breathing was included after the second cycle. To assess subject compliance and motion, we monitored real-time respiration signals using respiratory bellows throughout the scan. Any patients observed to deviate from the instructions (ie, unable to breath-hold and/or breathe normally when instructed by the research technologist or observed to move excessively) were flagged as "noncompliant."

\section{Breath-Hold CVR and MR Imaging Acquisition}

Children underwent repeat BH-CVR studies (1A and $1 \mathrm{~B}$ ) on the same day during the same MR imaging session. Follow-up repeat studies were performed in a subset of patients at later time-points (2A-2B; 3A-3B; 4A-4B).

MR imaging data were acquired with a 3T scanner (Achieva; Philips Healthcare, Best, the Netherlands) using an 8-channel head coil. The BH-CVR protocol consisted of 2 separate BOLD acquisitions using EPI-gradient recalled echo lasting 6 minutes, 6 seconds $(25$ slices; $\mathrm{TR} / \mathrm{TE}=2000 / 30 \mathrm{~ms}$; voxel size $=3.4 \times 3.4 \times$ $5 \mathrm{~mm}^{3} ; \mathrm{FOV}=22 \mathrm{~cm} ; 180$ dynamics $)$. The $\mathrm{BH}$ paradigm was used throughout the scan duration. A high-resolution 3D T1-weighted structural image $\left(160\right.$ slices; voxel size $=0.86 \times 0.86 \times 1 \mathrm{~mm}^{3}$; $\mathrm{FOV}=22 \mathrm{~cm}$ ) was acquired for tissue classification and coregistering the CVR maps.

\section{Image Postprocessing and CVR Estimation}

MR imaging data processing was performed using functions from Analysis of Functional Neuro Images (AFNI, Version 16.1.04; http://afni.nimh.nih.gov/afni) ${ }^{23}$ and FSL (Version 4.1.9; (http:// www.fmrib.ox.ac.uk/fsl) toolboxes. The first 2 dynamics of the BOLD data were truncated and subjected to slice-timing correction. Dynamics were corrected for motion by including the 6 


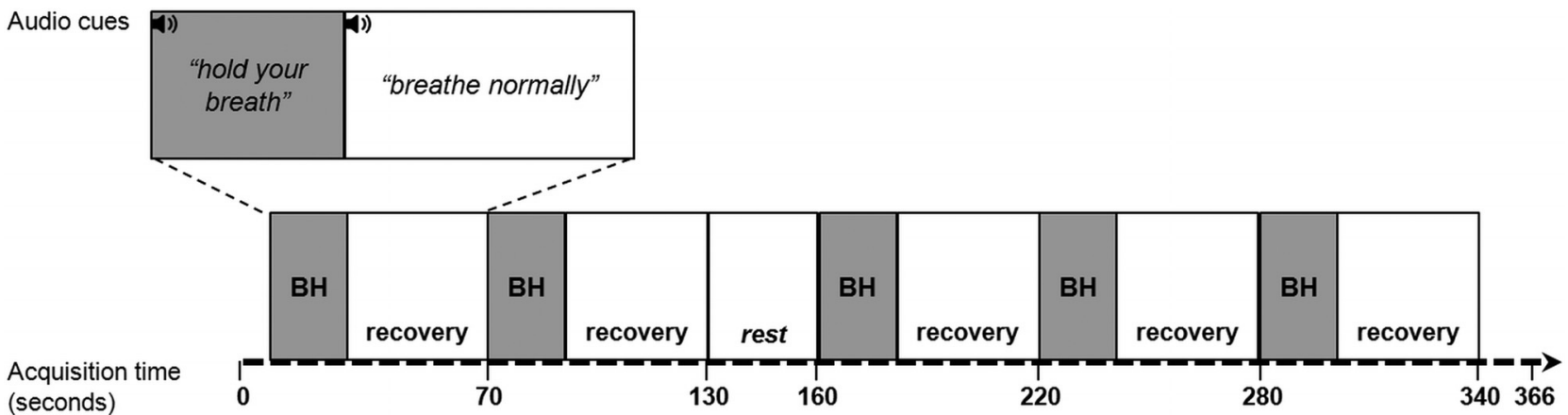

A

Averaged signal from the cerebellum

B

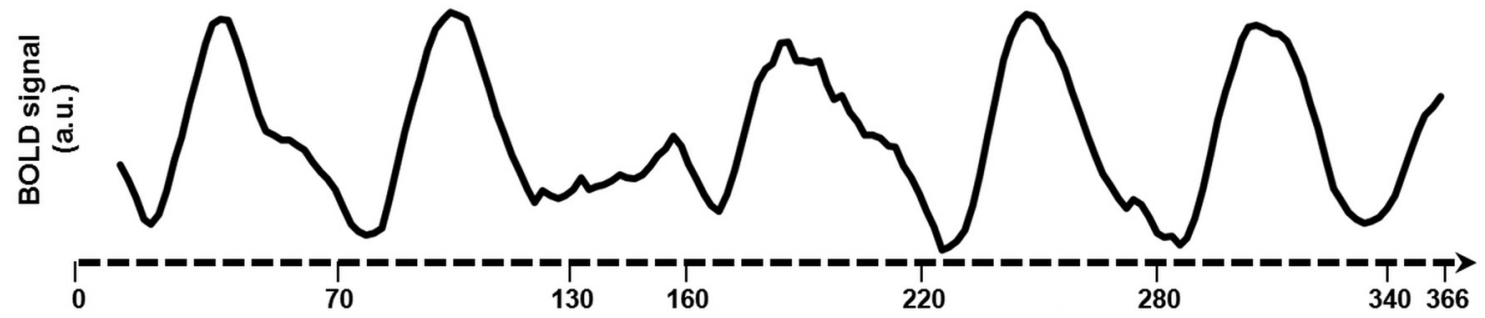

FIG 1. Schematic of breath-hold paradigm (A) and cerebellar BOLD signal time course $(B)$ obtained from a representative participant (P11). a.u. indicates arbitrary units.

rigid-body-movement parameters as covariates in a generalized linear model. Spatial deviations with respect to a reference dynamic were also computed. BOLD dynamics exhibiting excessive motion ( $>0.3 \mathrm{~mm} /$ dynamic) were automatically labeled and disregarded in subsequent analyses. Data were spatially smoothed using a Gaussian kernel of $7 \mathrm{~mm}$. The Automated Segmentation Tool of FSL (FAST; http://fsl.fmrib.ox.ac.uk/fsl/fslwiki/fast) was used for defining GM and WM masks. ${ }^{24}$ The mask of the cerebellum from the Montreal Neurological Institute 152 atlas was coregistered to each patient to determine the cerebellar region.

For CVR maps, the patient's BOLD time-series in each voxel of the brain was subjected to generalized linear model analysis, using the corresponding averaged cerebellar time courses as a regressor (Fig $1 B$ ). The regression coefficients (or the $\beta$ weights) were then calculated for each voxel. Negative $\beta$ weights describing an inverse relationship with the regressor are the markers of steal. CVR maps consisting of voxelwise negative and positive $\beta$ weights (describing a negative and positive relationship with the regressor, respectively) were coregistered to the high-resolution $\mathrm{T} 1$ images in the native space for visualization. ${ }^{25}$ A combined CVR measure was also computed as a weighted average of relative counts of positive and negative voxels. ${ }^{19}$ In addition, all CVR measures were summarized by major vascular territory, namely the middle cerebral artery, anterior cerebral artery, and posterior cerebral artery, defined using the regions in the Harvard-Oxford Atlas. ${ }^{26-29}$

\section{Assessment of BH-CVR Performance}

The quality of $\mathrm{BH}$ studies was assessed using the following: 1) $\mathrm{BH}$ compliance during data acquisition using a respiratory waveform from the bellows, and 2) evidence of excessive motion during postprocessing. The determination was based on a cutoff set at $>15$ dynamics, or 30 seconds, affected by motion in either $\mathrm{BH}$ repeat study.
Criteria for scoring CVR maps

\begin{tabular}{lc}
\hline \multicolumn{1}{c}{ Visual Impression } & Score \\
\hline Normal CVR & 1 \\
Reduced positive reactivity \pm minimal steal $(<10 \%)$ & 2 \\
Significant steal $(>10 \%)$ & 3 \\
\hline
\end{tabular}

\section{Qualitative Scoring of BH-CVR Maps}

Summative scoring of the ROIs (whole-brain and hemispheric vascular territories) of the BH-CVR maps was conducted by visual inspection for steal of the first repeat study $(1 \mathrm{~A}-1 \mathrm{~B})$ by neurologists, blinded to the patients' clinical information. Negative CVR reactivity observed in CSF-rich ventricular regions, including the peritrigonal regions, has been reported in healthy individuals. ${ }^{30}$ It is physiologic and hence considered an artifact in CVR interpretation. ${ }^{31}$ The visual impression of normal positive reactivity, reduced positive reactivity \pm minimal steal, and significant $(>10 \%$ of the ROI) steal was scored as 1,2 , and 3, respectively (Table). Hemispheric scoring by visual inspection was conducted independently by the same neurologists for patients with multiple time point studies (1A-1B; $2 \mathrm{~A}-2 \mathrm{~B} ; 3 \mathrm{~A}-3 \mathrm{~B}$; $4 \mathrm{~A}-4 \mathrm{~B})$ (Fig 2).

\section{Moyamoya Subtype, Stroke, and Intellectual Function}

Clinical records and high-resolution clinical T2-weighted images acquired at the time of each BH-CVR study were reviewed for Moyamoya subtype, history of stroke, and the presence of vascular territory ischemic infarction.

Standardized age-appropriate measures of intelligence using the Wechsler Intelligence Scale for Children (IV/V), ${ }^{26,27}$ administered as part of routine clinical care within 3 years of $\mathrm{BH}-\mathrm{CVR}$ collection, were reviewed and included in the analysis. 


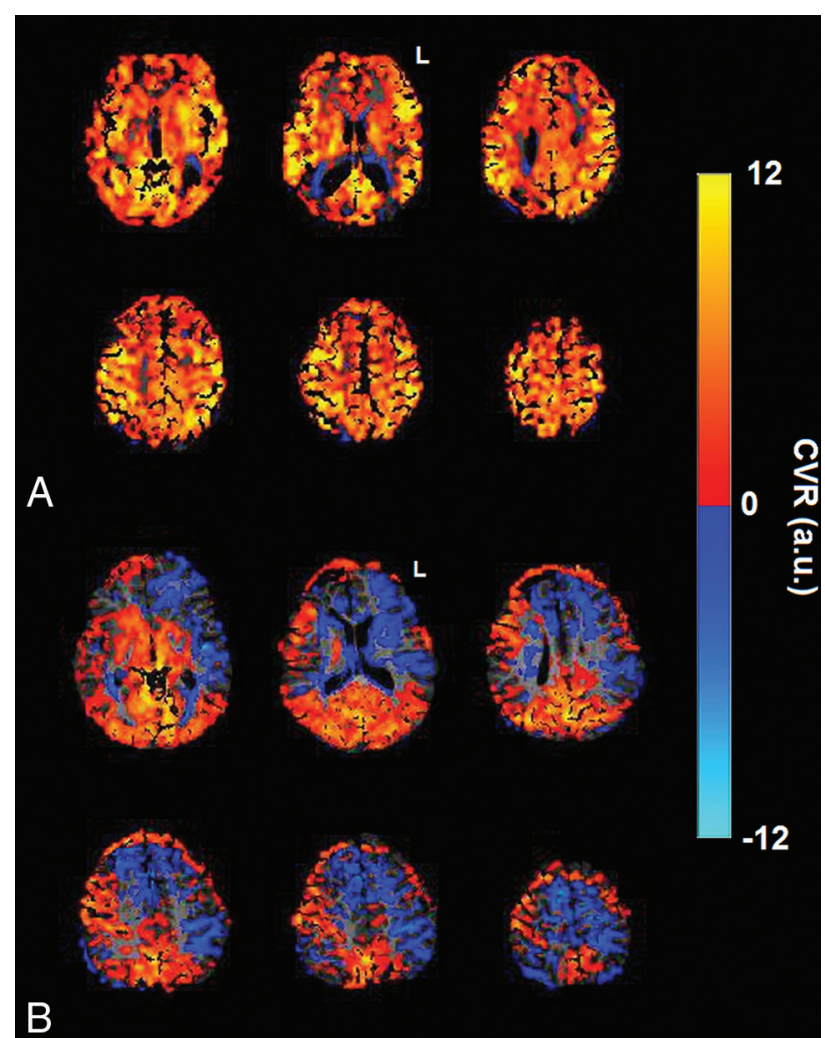

FIG 2. A, Normal-appearing BH-CVR maps demonstrating positive $\mathrm{BH}-\mathrm{CVR}$ reactivity (P07: whole-brain score, 1 ; hemispheric score, 1:1). $B$, $\mathrm{BH}-\mathrm{CVR}$ maps with abnormal findings demonstrating bilateral abnormal (left > right) negative reactivity (P15: whole-brain score, 3; hemispheric score, 3:3). L indicates left; a.u., arbitrary units.

\section{Statistical Analysis}

To determine the repeatability, we computed the ICC and coefficient of variation $(\mathrm{CV})$ of within-day repeat scans in the whole brain and in tissue (GM, WM) masks. ${ }^{21} \mathrm{CV}$ was computed as a ratio of the SD and mean of CVR estimates among repeat studies. Interpretation of ICC values was as follows: $<0.41$, "poor"; $\geq 0.41$ and $<0.59$, "fair"; $\geq 0.59$ and $<0.74$, "good"; and $\geq 0.74$, "excellent." 32 Bland-Altman analysis was performed to determine the 95\% limits of agreement among the repeat BH-CVR estimates. The Cohen $\kappa$ analysis was used to assess the following: 1) agreement among whole-brain scores, 2) agreement among scores by vascular territory, and 3) interrater reliability of hemispheric scoring of repeat scans. Interpretation of the $\kappa$ scores was as follows: 0.01, "poor"; 0.01-0.20, "slight"; 0.21-0.40, "fair"; 0.410.60 , "moderate"; 0.61-0.80, "substantial"; and 0.81-1.00, “almost perfect" agreement. ${ }^{33}$

Means of positive, negative, and combined CVR estimates, representing the magnitude of CVR values, and their respective voxel counts were computed and compared among repeat scans using paired $t$ tests. These estimates were also compared among repeat scans in the left and right hemispheric vascular territories, using 3-way repeat-measures ANOVA.

Exploratory analyses to examine relationships among ICC, CVR estimates, clinical factors (Moyamoya subtype, history of stroke), demographics (age at CVR), and intellectual function (full-scale intelligence quotient and intelligence quotient subscales) were conducted. One-way ANOVA was used for categoric variables; and linear regression, for continuous independent variables. Post hoc $t$ tests were performed to assess significant ANOVA findings. Fisher exact tests were used for comparing 2 categoric variables.

\section{RESULTS}

\section{Study Population}

Twenty children ( 11 males; mean age, $12.1 \pm 3.3$ years; median, 11.7 years; range, $6.2-18.0$ years) at the first $\mathrm{BH}-\mathrm{CVR}$ were included. Follow-up repeat scans (2A-2B) were collected in 6 children, 3 children returned for a third scan $(3 \mathrm{~A}-3 \mathrm{~B})$, and 1 child returned for a fourth scan $(4 \mathrm{~A}-4 \mathrm{~B})$. Thirty pairs of within-day repeat $\mathrm{BH}-\mathrm{CVR}$ studies (60 $\mathrm{BH}-\mathrm{CVR}$ scans) were completed.

Eleven patients (55\%) had idiopathic Moyamoya disease, 4 (20\%) had neurofibromatosis type 1, two (10\%) had SCD, while 3 others (15\%) had familial, radiation-induced, or ACTA2 mutation Moyamoya disease (On-line Table 1); the mean age at Moyamoya disease diagnosis was $7.9 \pm 3.2$ years (median, 8.0 years; range, 1.4-16.8 years). Three patients (15\%) presented with stroke, and 7 patients (35\%), with TIA. Seventeen patients (85\%) had no prior history of stroke. Half of the children were either asymptomatic $(7 / 20,35 \%)$ or had headache only $(3 / 20,15 \%)$ at the time of Moyamoya disease diagnosis.

\section{BH Performance, CVR Estimates, and Repeatability Measures}

In 6 of 30 studies (20\%), the children were flagged as noncompliant, all of whom were undergoing first repeat studies (1A or 1B). Four of those were noted to be noncompliant during data acquisition. Two studies had excessive motion noted during postprocessing. All studies irrespective of compliance or motion were included in the assessment of repeatability (On-line Table 1).

Mean $\mathrm{BH}$ durations in the first (A) and the second (B) scans were $19.6 \pm 3.3$ seconds (range, 12-25 seconds) and $20.3 \pm 3.4$ seconds (range, $14-25$ seconds), respectively $(P=.1$; median for $A$ and $B=20$ seconds). No significant differences between repeat scans were found for the positive, negative, or combined mean CVR estimates or the counts of positive and negative voxels in the GM and the WM (all $P>$.05; patient-wise estimates in On-line Table 2). The CVR estimates differed across the vascular territories but, most important, not among repeat scans (all $P>.05$; On-line Fig 1). All estimates differed between the anterior cerebral artery and posterior cerebral artery and MCA and posterior cerebral artery territories, with no difference between the anterior cerebral artery and MCA $(P<.05$, Bonferroni-corrected). Of 30 paired studies, repeatability for the whole-brain CVR estimates $(\mathrm{ICC}=0.703 \pm 0.190)$ was excellent in $18(60 \%)$ and good in 5 (17\%) studies. The remaining 7 studies $(23 \%)$ fell into the fairto-poor range (On-line Table 1). All except 1 (5/6) patient flagged as either noncompliant or for excessive motion had poor ICCs. The 2 patients with SCD also exhibited relatively low ICCs (0.51$0.48)$. The ICC tended to vary across vascular territories $(P=.07)$ but fell in the good range $(\mathrm{MCA}=0.67 \pm 0.16$; anterior cerebral artery $=0.65 \pm 0.19$; posterior cerebral artery $=0.71 \pm 0.12$; On-line Fig 2).

The mean CV was $9.1 \% \pm 8.2 \%$ for positive CVR and $22.5 \% \pm$ $18.3 \%$ for negative CVR in the GM. CV was reduced, on average, 


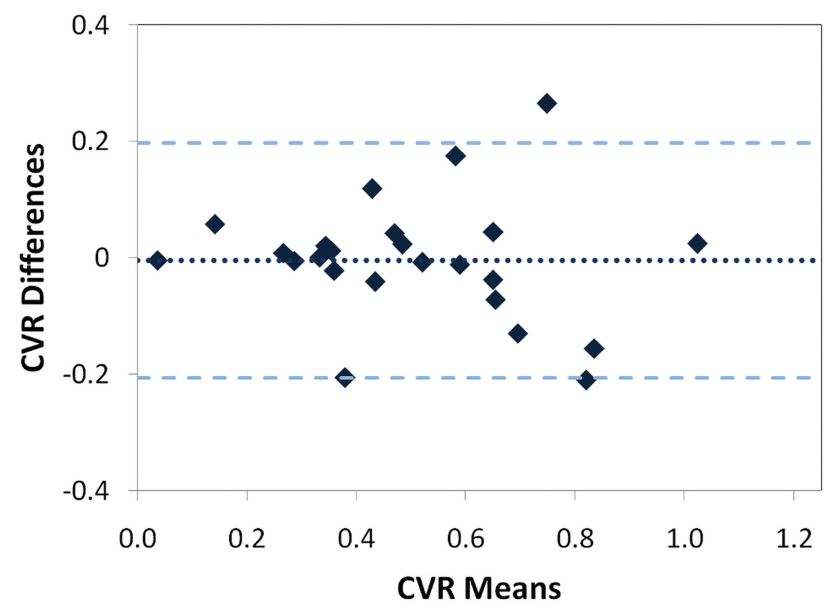

FIG 3. Bland-Altman plot showing a low mean between-scan difference of 0.004 (dotted line) with a critical difference of 0.202 (broken lines).

by $2.5 \%$ after excluding the studies flagged for noncompliance. $\mathrm{CV}$ and ICC were negatively correlated $(P<.05)$. A Bland-Altman plot (Fig 3) illustrated the overall variation of whole-brain CVR with a test-retest difference of $-0.004 \pm 0.202$.

\section{Qualitative Scoring by Visual Inspection}

Three (1A or 1B) repeat studies were excluded as not being suitable for scoring by visual inspection. In the remaining paired studies (17/20), there was $82.35 \%(14 / 17)$ agreement in wholebrain (1A-1B) scores. The Cohen weighted $\kappa$ was 0.75 , in keeping with good strength of agreement. The Cohen weighted $\kappa$ by vascular territory was 0.95 (MCA), 0.8 (anterior cerebral artery), and 1.0 (posterior cerebral artery), suggestive of almost perfect strength of agreement. The interrater reliability for hemispheric scoring was 1.0 (left) and 0.83 (right), suggestive of perfect and very good strength of agreement among scorers, respectively.

\section{Age, Moyamoya Subtype, Stroke, Intellectual Function, and Measures of BH-CVR Repeatability}

Neither Moyamoya subtype nor a history of stroke was associated with ICC ranks (excellent, fair/good, poor; $P>.05$ ). A significant effect of age at the time of BH-CVR on the ICC ranks was found (mean age in poor, excellent, and good ICC groups, $8.6 \pm 1.7$, $13.1 \pm 2.6$, and $14.2 \pm 2.9$ years, respectively; $F(2,20)=4.3 ; P=$ .027). The ICC was not associated with the full-scale intelligence quotient $(n=9)$ or subscales $(P>.05)$. However, the count of negative CVR voxels was a significant predictor of the processingspeed index (GM, $P=.015$; WM, $P=.047)$ after controlling for $\mathrm{BH}$ duration and age. In addition, the processing-speed index was significantly reduced in patients with whole-brain qualitative scores of 3 (ie, in patients exhibiting significant steal) $(P=.009)$.

\section{DISCUSSION}

Our study demonstrates that BH-CVR implemented using standard functional MR images provides a feasible, repeatable, and reliably interpretable tool for the assessment of CVR and cerebrovascular reserve in children with Moyamoya disease. On average, children older than 8.6 years, composing $80 \%$ of our cohort, had no difficulty with our $\mathrm{BH}$ paradigm. In this group, within-day repeatability was good-to-excellent, CV was $<25 \%$, and agreement of repeat measures was acceptable. Repeatability was not affected by clinical factors such as Moyamoya disease etiology, history of stroke, or intellectual abilities. Negative CVR or steal, however, was associated with a reduced processing-speed index in a subset of our cohort. Another important goal of our study was to explore whether BH-CVR maps are reliably interpretable among different clinical users. We found good-to-excellent agreement of within-day visual inspection scores and almost perfect interrater agreement.

A significant number of children with Moyamoya disease are asymptomatic at the time of diagnosis and therefore require lifelong surveillance. This was reflected in our study in which $35 \%$ of our patients were asymptomatic at diagnosis. Our study also included the youngest child with Moyamoya disease reported to date to successfully complete BH-CVR studies (6.2 years of age). ${ }^{20,21,30}$ Lowering the age limit of feasibility to younger than 7 years for obtaining the CVR measures will facilitate earlier instigation of longitudinal surveillance in Moyamoya disease than is the current practice and will inform clinical management in the age group at highest risk of stroke.

A number of factors affect the BOLD response and thereby measures of repeatability. This was highlighted by our 2 patients with SCD who had notably lower repeatability, despite completing the $\mathrm{BH}-\mathrm{CVR}$ without any issues with compliance or motion. This result may be attributed to the anemia in SCD driving a global impairment in $\mathrm{CVR}^{34,35}$ and reducing the overall signalto-noise ratio of the BOLD response. The increased influence of noise can adversely affect the correlation of repeat scans. In addition, negative CVR, which is frequently seen in SCD, ${ }^{35}$ is especially prone to high variability and therefore can affect repeatability in these patients, as suggested by our data.

The $\mathrm{BH}$ execution and the duration of $\mathrm{BH}$ can also affect the $\mathrm{BOLD}$ response. A short $\mathrm{BH}$ of 3 seconds is adequate to produce $\mathrm{a}$ detectable BOLD response. However, a longer $\mathrm{BH}$ increases the magnitude and number of voxels exhibiting the response, resulting in more robust and repeatable measures of BOLD-CVR. ${ }^{36}$ The mean $\mathrm{BH}$ duration in our study ranged from 19.6 to 20.3 seconds, suggestive of good $\mathrm{BH}$ duration overall. One of the limitations of the $\mathrm{BH}$ challenge is that it is limited to patients who can understand and comply with instructions. It therefore does not adequately address the challenge of conducting CVRs in the very young (eg, younger than 5 years of age). However, supervised practice of the paradigm and use of good $\mathrm{BH}$ practice guidelines such as paced breathing between challenges and breath-holding after expiration both at home and outside the MR imaging scanner before the BH-CVR study may help improve $\mathrm{BH}$ performance, reduce variability, and, in turn, improve repeatability. ${ }^{22}$ Other strategies to minimize motion during scanning include measures to reduce distractibility among BH-CVR cycles such as visual fixation with videos and/or games.

Real-time monitoring of the carbon dioxide stimulus is the desirable method for CVR quantification. ${ }^{22}$ Alternatively, as in our study, the dynamic cerebellar BOLD time course can be used. ${ }^{37}$ In this method, the BOLD signal from the cerebellum represents changes in the blood flow corresponding to the accumulation of arterial carbon dioxide in each $\mathrm{BH}$ and provides a 
dynamic trace reference for quantifying BOLD in the rest of the brain tissue. While this method is reliable for within-subject assessment of CVR, a limitation is that it cannot be used for group comparative analysis. The use of MR imaging-compatible technologies for real-time acquisition of carbon dioxide in conjunction with BOLD and $\mathrm{BH}$ will facilitate group-based and more sophisticated quantitative analysis in future studies.

\section{CONCLUSIONS}

Measures of BH-CVR repeatability in children are good-to-excellent and CVR maps are reliably interpretable by clinical users. While clinical acquisition of MR imaging-CVR using computercontrolled devices has become increasingly feasible with recent iterations of this technology, the relative simplicity of BH-CVR is desirable for younger patients who can follow simple instructions. In addition, $\mathrm{BH}-\mathrm{CVR}$ remains a practical substitute for many institutions when controlled-delivery carbon dioxide methods are not available or tolerable. Standardization of $\mathrm{BH}$ paradigms, image acquisition, and processing protocols will further allow the implementation of this promising technique for clinical assessment of cerebrovascular reserve and ischemic risk in childhood cerebrovascular disease.

\section{ACKNOWLEDGMENTS}

We acknowledge the support of The Auxillium Foundation, the Stroke Imaging Laboratory for Children, and the Ontasian Imaging Laboratory.

Disclosures: Fenella Kirkham-UNRELATED: Employment: University College London Great Ormond Street Institute of Child Health; Grants/Grants Pending: National Institute of Health Research (UK), Actione Medical Research; Payment for Lectures Including Service on Speakers Bureaus: Fenella Kirkham; Payment for Manuscript Preparation: Shire plc Pharmaceutical Company, Paid to Fenella Kirkham; Comments: for epilepsy; Royalties: Mac Keith Press, Comments: small amount for previously published book on childhood stroke; Travel/Accommodations/Meeting Expenses Unrelated to Activities Listed: European Haemoglobinopathy Association, Comments: travel to Madrid and Cairo to give lectures.

\section{REFERENCES}

1. Lynch JK, Hirtz DG, DeVeber G, et al. Report of the National Institute of Neurological Disorders and Stroke workshop on perinatal and childhood stroke. Pediatrics 2002;109:116-23 CrossRef Medline

2. Fullerton HJ, Wu YW, Zhao S, et al. Risk of stroke in children: ethnic and gender disparities. Neurology 2003;61:189-94 CrossRef Medline

3. Fullerton HJ, Wu YW, Sidney S, et al. Risk of recurrent childhood arterial ischemic stroke in a population-based cohort: the importance of cerebrovascular imaging. Pediatrics 2007;119:495-501 CrossRef Medline

4. Mackay MT, Wiznitzer M, Benedict SL, et al; International Pediatric Stroke Study Group. Arterial ischemic stroke risk factors: the International Pediatric Stroke Study. Ann Neurol 2011;69:130-40 CrossRef Medline

5. Wintermark M, Hills NK, deVeber GA, et al; VIPS Investigators. Arteriopathy diagnosis in childhood arterial ischemic stroke: results of the vascular effects of infection in pediatric stroke study. Stroke 2014;45:3597-605 CrossRef Medline

6. Ganesan V, Prengler M, Wade A, et al. Clinical and radiological recurrence after childhood arterial ischemic stroke. Circulation 2006;114:2170-77 CrossRef Medline

7. Braun KP, Bulder MM, Chabrier S, et al. The course and outcome of unilateral intracranial arteriopathy in 79 children with ischaemic stroke. Brain 2009;132:544-57 Medline

8. Amlie-Lefond C, Bernard TJ, Sebire G, et al; International Pediatric
Stroke Study Group. Predictors of cerebral arteriopathy in children with arterial ischemic stroke: results of the International Pediatric Stroke Study. Circulation 2009;119:1417-23 CrossRef Medline

9. Dobson SR, Holden KR, Nietert PJ, et al. Moyamoya syndrome in childhood sickle cell disease: a predictive factor for recurrent cerebrovascular events. Blood 2002;99:3144-50 CrossRef Medline

10. Rafay MF, Armstrong D, Dirks P, et al. Patterns of cerebral ischemia in children with moyamoya. Pediatr Neurol 2015;52:65-72 CrossRef Medline

11. Guey S, Tournier-Lasserve E, Hervé D, et al. Moyamoya disease and syndromes: from genetics to clinical management. Appl Clin Genet 2015;8:49-68 CrossRef Medline

12. Sobczyk O, Battisti-Charbonney A, Fierstra J, et al. A conceptual model for $\mathrm{CO}_{2}$-induced redistribution of cerebral blood flow with experimental confirmation using BOLD MRI. Neuroimage 2014;92: 56-68 CrossRef Medline

13. Gupta A, Chazen JL, Hartman M, et al. Cerebrovascular reserve and stroke risk in patients with carotid stenosis or occlusion: a systematic review and meta-analysis. Stroke 2012;43:2884-91 CrossRef Medline

14. Conklin J, Fierstra J, Crawley AP, et al. Impaired cerebrovascular reactivity with steal phenomenon is associated with increased diffusion in white matter of patients with Moyamoya disease. Stroke 2010;41:1610-16 CrossRef Medline

15. Reinhard M, Schwarzer G, Briel M, et al. Cerebrovascular reactivity predicts stroke in high-grade carotid artery disease. Neurology 2014; 83:1424-31 CrossRef Medline

16. Lee M, Zaharchuk G, Guzman R, et al. Quantitative hemodynamic studies in moyamoya disease: a review. Neurosurg Focus 2009;26:E5 CrossRef Medline

17. Slessarev M, Han J, Mardimae A, et al. Prospective targeting and control of end-tidal CO2 and O2 concentrations. J Physiol 2007;581: 1207-19 CrossRef Medline

18. Kassner A, Winter JD, Poublanc J, et al. Blood-oxygen level dependent MRI measures of cerebrovascular reactivity using a controlled respiratory challenge: reproducibility and gender differences. $J$ Magn Reson Imaging 2010;31:298-304 CrossRef Medline

19. Heyn C, Poublanc J, Crawley A, et al. Quantification of cerebrovascular reactivity by blood oxygen level-dependent MR imaging and correlation with conventional angiography in patients with Moyamoya disease. AJNR Am J Neuroradiol 2010;31:862-67 CrossRef Medline

20. Han JS, Mikulis DJ, Mardimae A, et al. Measurement of cerebrovascular reactivity in pediatric patients with cerebral vasculopathy using blood oxygen level-dependent MRI. Stroke 2011;42:1261-69 CrossRef Medline

21. Leung J, Kim JA, Kassner A. Reproducibility of cerebrovascular reactivity measures in children using BOLD MRI. J Magn Reson Imaging 2016;43:1191-95 CrossRef Medline

22. Bright MG, Murphy K. Reliable quantification of BOLD fMRI cerebrovascular reactivity despite poor breath-hold performance. $\mathrm{Neu}$ roimage 2013;83:559-68 CrossRef Medline

23. Fukui M. Guidelines for the diagnosis and treatment of spontaneous occlusion of the circle of Willis ('moyamoya' disease): Research Committee on Spontaneous Occlusion of the Circle of Willis (Moyamoya Disease) of the Ministry of Health and Welfare, Japan. Clin Neurol Neurosurg 1997;99(Suppl 2):S238-40 Medline

24. Zhang Y, Brady M, Smith S. Segmentation of brain MR images through a hidden Markov random field model and the expectationmaximization algorithm. IEEE Trans Med Imaging 2001;20:45-57 CrossRef Medline

25. Jenkinson M, Smith S. A global optimisation method for robust affine registration of brain images. Med Image Anal 2001;5:143-56 CrossRef Medline

26. Wechsler D. Wechsler Intelligence Scale for Children: Fourth Edition Technical and Interpretive Manual. San Antonio: PsychCorp; 2004

27. Wechsler D. Wechsler Intelligence Scale for Children: Fifth Edition. 
Technical and Interpretive Manual. Bloomington: PsychCorp: Pearson; 2014

28. van der Zwan A, Hillen B, Tulleken CA, et al. Variability of the territories of the major cerebral arteries. J Neurosurg 1992;77:927-40 CrossRef Medline

29. Desikan RS, Ségonne F, Fischl B, et al. An automated labeling system for subdividing the human cerebral cortex on MRI scans into gyral based regions of interest. Neuroimage 2006;31:968-80 CrossRef Medline

30. Mandell DM, Han JS, Poublanc J, et al. Mapping cerebrovascular reactivity using blood oxygen level-dependent MRI in patients with arterial steno-occlusive disease: comparison with arterial spin labeling MRI. Stroke 2008;39:2021-28 CrossRef Medline

31. Thomas BP, Liu P, Aslan S, et al. Physiologic underpinnings of negative BOLD cerebrovascular reactivity in brain ventricles. Neuroimage 2013;83:505-12 CrossRef Medline

32. Lipp I, Murphy K, Caseras X, et al. Agreement and repeatability of vascular reactivity estimates based on a breath-hold task and a resting state scan. Neuroimage 2015;113:387-96 CrossRef Medline

33. Landis JR, Koch GG. The measurement of observer agreement for categorical data. Biometrics 1977;33:159-74 CrossRef Medline

34. Kosinski PD, Croal PL, Leung J, et al. The severity of anaemia depletes cerebrovascular dilatory reserve in children with sickle cell disease: a quantitative magnetic resonance imaging study. $\mathrm{Br} \mathrm{J}$ Haematol 2017;176:280-87 CrossRef Medline

35. Prohovnik I, Hurlet-Jensen A, Adams R, et al. Hemodynamic etiology of elevated flow velocity and stroke in sickle-cell disease. J Cereb Blood Flow Metab 2009;29:803-10 CrossRef Medline

36. Abbott DF, Opdam HI, Briellmann RS, et al. Brief breath holding may confound functional magnetic resonance imaging studies. Hum Brain Mapp 2005;24:284-90 CrossRef Medline

37. Raut RV, Nair VA, Sattin JA, et al. Hypercapnic evaluation of vascular reactivity in healthy aging and acute stroke via functional MRI. Neuroimage Clin 2016;12:173-79 CrossRef Medline 